\title{
THEORETICAL AND OBSERVED DISTANCE CORRECTIONS FOR RAYLEIGH-WAVE MAGNITUDE
}

\author{
By R. W. Alewine, III
}

\begin{abstract}
Examination of the distance correction factor used in the widely accepted formula for surface-wave magnitude reveals that this empirically derived linear formula fails to give an accurate approximation to the theoretical nonlinear amplitude-distance relation for epicentral distances less than $15^{\circ}$. For epicentral distances greater than $15^{\circ}$, the empirical formula contains an implied oceanic-type energy-dissipation coefficient. When the original Gutenberg theoretical surfacewave magnitude formula with an appropriate continental energy-dissipation coefficient is applied to explosion data from the Nevada Test Site, a consistent surface-wave magnitude is obtained at all distances. A systematic method of normalizing Rayleigh-wave magnitudes obtained over different types of propagation paths is suggested. This normalization might provide a means for better separating natural events and explosions in the $m_{b}-M_{s}$ plots.
\end{abstract}

\section{INTRODUCTION}

Recent investigations of Rayleigh-wave magnitude from explosions and earthquakes in continental areas have indicated distance-dependent magnitudes when the usual Gutenberg-Richter (1936) surface-wave magnitude formula is applied. In order to eliminate this magnitude dependence on distance, Evernden (1971), Evernden et al.,(1971), and Basham (1971), using long-period records from Long Range Seismic Measurements (LRSM) stations in the United States and permanent stations in Canada, have proposed new distance power dependences which are lower than those used in the generally accepted amplitude versus distance expression used in determining magnitudes. These new power dependences are similar to those reported by Taner (1961) for shallowfocus earthquakes recorded at small epicentral distances. Distance-independent magnitudes are essential in the determination of accurate and consistent surface-wave magnitudes at short to intermediate distances. This accurate information is needed in order to lower the threshold of discrimination between underground explosions and shallow earthquakes. Also, some means is necessary to systematically compare magnitudes measured at a single station from different events with different crustal and uppermantle structure along the propagation path. Conversely, the removal of path effects would allow a better comparison of magnitudes obtained at different stations for the same event.

Comparison of empirical and theoretical distance corrections. A critical examination of the formulation of the original surface-wave magnitude scale reveals that the distance correction term used in the standard formula given by Gutenberg and Richter (1936) is not intended to give the needed accuracy at these short to intermediate distances for events with purely continental propagation paths. This standard formula is given by

$$
M_{s}=\log _{10} A_{H}-\log _{10} B^{E}
$$

where $A_{H}$ is the maximum zero-to-peak amplitude in microns of the horizontal component of the fundamental-mode Rayleigh wave having a period of about $20 \mathrm{sec}$, and $-\log B^{E}$ is a correction factor that is given as a function of distance. The quantity 
$-\log _{10} B^{E}$ is an empirical relation given either in tabular form as in Gutenberg (1945) and Richter (1958), or can be expressed as

$$
-\log _{10} B^{E}=1.656 \log _{10} \Delta+1.818
$$

where $\Delta$ is the epicentral distance in degrees confined to lie between $15^{\circ}$ and $130^{\circ}$.

For maximum amplitudes having periods different from $20 \mathrm{sec}$, this formula has been modified to approximate the amplitude of the 20-sec wave by

$$
M_{s}=\log _{10}\left(\frac{A_{H}}{T}\right)+1.656 \log _{10} \Delta+3.119
$$

This last formula is equivalent to within an additive constant of 0.181 of the Prague 1962 formula given by Vanek et al., (1962)

$$
M_{s}^{P r}=\log _{10}\left(\frac{A_{H}}{T}\right)+1.656 \log _{10} \Delta+3.3 .
$$

A slight variation to the Prague formula by which the vertical component of the Rayleigh waves is used to avoid contamination with Love waves is in general use in the United States and Canada. This latter formula should yield magnitudes greater than those given in (4) by the constant value, $\log \left(1 / \varepsilon_{0}\right), \varepsilon_{o}$ being the surface ellipicity of the period of the Rayleigh wave used. For a typical continental model, waves having periods in the range of 8 to $25 \mathrm{sec}$ yield a value of about 0.17 .

However, it must be emphasized that the $-\log B^{E}$ factor given in (2) and subsequently used in (3) and (4) is an empirical relation found by Gutenberg (1945) by applying a least-squares estimate to observed amplitude data obtained primarily from earthquakes around the Pacific and recorded in Pasadena. In performing this least-squares fit, Gutenberg found that for those events with epicentral distances between $15^{\circ}$ and $130^{\circ}$, a plot of the amplitudes versus the logarithm of the distance yielded nearly a linear relation. The $-\log B^{E}$ given in (2) was adopted as a linear approximation over a particular range to the nonlinear theoretical distance correction term discussed below. Thus, the $-\log B^{E}$ correction term is based simply on a best fit of data from events having primarily oceanic travel paths in the $15^{\circ}$ to $130^{\circ}$ distance range.

Basham (1971) and Evernden (1971) plotted the variation of Rayleigh-wave amplitude with distance for short to intermediate continental ranges and performed a linear leastsquares fit to the observed data. The coefficients of the $\log _{10} \Delta$ term that they present are smaller than that given by Gutenberg (1945) over longer oceanic ranges. With these lower power dependencies, the theoretical formula can be approximated by a linear relation over the specified range interval and type of propagation path. Nuttli (1972) gives a thorough discussion of these limited-range linear approximations to the theoretical nonlinear formula.

Gutenberg and Richter (1936) give the following theoretical expression for the distance absorption effect for waves having periods near the minimum in the group-velocity dispersion curve.

$$
\frac{A_{2}}{T_{2}}=\frac{A_{1}}{T_{1}} \exp \left[-(k / 2)\left(\Delta_{2}-\Delta_{1}\right) 111.2\right]\left[\frac{\operatorname{Sin} \Delta_{1}}{\operatorname{Sin} \Delta_{2}}\right]^{1 / 2}\left[\frac{\Delta_{1}}{\Delta_{2}}\right]^{1 / 6}
$$

where $A_{n}$ are amplitudes, taken to be the horizontal amplitudes in microns, $T_{n}$ are periods at some distance $\Delta_{n}$, expressed in degrees, and $k$ is the coefficient of energy absorption, expressed in inverse kilometers, at the period $T_{n}$. It is to be noted that this expression contains a $(r)^{-1 / 2}$ geometrical spreading factor and an amplitude decay term owing to 
dispersion which goes as $(r)^{-1 / 6}$. However, Ewing et al., (1957) report that the amplitude variation owing to dispersion alone should go as $(r)^{-1 / 3}$. This effect will be discussed in more detail in a separate paper in preparation (Alewine, 1972). In all of the calculations in this paper, we will follow Gutenberg and Richter (1936) in approximating this amplitude decay as $(r)^{-1 / 6}$.

The absorption coefficient, $k$, is a function of the type of path traversed by the wave as it propagates from point 1 to point 2 . Now if we set $\Delta_{1}$ to be $90^{\circ}, T_{1}$ to be $20 \mathrm{sec}$, and define

then we obtain

$$
C=\exp \left[-(k / 2)\left(\Delta_{2}-\Delta_{1}\right) 111.2\right]\left[\frac{\operatorname{Sin} \Delta_{1}}{\operatorname{Sin} \Delta_{2}}\right]^{1 / 2}\left[\frac{\Delta_{1}}{\Delta_{2}}\right]^{1 / 6}
$$

where

$$
\log _{10}\left[A\left(\Delta=90^{\circ}\right)\right]=\log _{10}\left[\frac{A_{2}}{T_{2}}\right]-\log _{10} C+\log _{10} 20
$$

$$
-\log _{10} C=\frac{1}{2}\left[48.25 k\left(\Delta_{2}-90^{\circ}\right)+\log _{10} \operatorname{Sin} \Delta_{2}+\frac{1}{3}\left(\log _{10} \Delta_{2}-1.954\right)\right] .
$$

But the surface-wave magnitude, $M_{s}$, is defined (Gutenberg and Richter, 1936) as

$$
M_{s}=\log _{10}\left[A_{H}\left(\Delta=90^{\circ}\right)\right]+5.04
$$

where the 5.04 was arbitrarily chosen to set the zero of the magnitude scale. Combining (7) and (9), we obtain for the theoretical surface-wave magnitude

$$
M_{s}=\log _{10}\left[\frac{A_{H}}{T}\right]-\log _{10} B^{T}+\log _{10}[20]
$$

where $-\log _{10} B^{T}=-\log _{10} C+5.04$

$$
-\log _{10} B^{T}=5.04+\frac{1}{2}\left[48.25 k\left(\Delta-90^{\circ}\right)+\log _{10} \operatorname{Sin} \Delta+\frac{1}{3}\left(\log _{10} \Delta-1.954\right)\right] .
$$

It is evident from (11) that the distance correction factor is not only a function of the epicentral distance but also of the energy-dissipation structure of the travel path. Gutenberg (1945) compared the values of $-\log B^{T}$, with an oceanic $k$, to $-\log B^{E}$ and found excellent agreement between the theoretical, empirical, and observed distancecorrection factors for distances greater than $30^{\circ}$ along an oceanic-type travel path. For distances less than $30^{\circ}$ the observed distance-correction factors are slightly smaller than those predicted by the theoretical equation, whereas the empirical factors are still quite close. In this comparison, Gutenberg used an oceanic $k$ value of $3.0 \times 10^{-4} \mathrm{~km}^{-1}$, which is about a factor of 2 greater than he observed for an average continental path. Data presented by Tsai and Aki (1969) comparing $Q$ values obtained from oceanic travel paths and continental travel paths seem to confirm this general ratio. Nuttli (1972) also finds that a dissipation coefficient of $2.74 \times 10^{-4} \mathrm{~km}^{-1}$ gives good theoretical agreement to the observed 20 -sec-period surface-wave amplitude-distance decay rate used in the formulation of the original surface-wave magnitude.

Thus, the empirical distance correction factor, (2), for distances greater than $15^{\circ}$ is essentially a good linear approximation to the theoretical factor, (11), with an oceanic dissipation structure. Because the magnitude scale has been used for events with worldwide travel paths, and the propagation path from the majority of the earthquakes to stations in the United States and Canada involves some oceanic-type structure, the $-\log B^{E}$ correction factor has performed quite adequately because most of the Earth's crust and upper-mantle structure is oceanic. However, the $-\log B^{E}$ term is not intended to accurately approximate $-\log B^{T}$ with a continental type $k$, or to correct properly the observed amplitude data for short, purely continental, travel paths. 


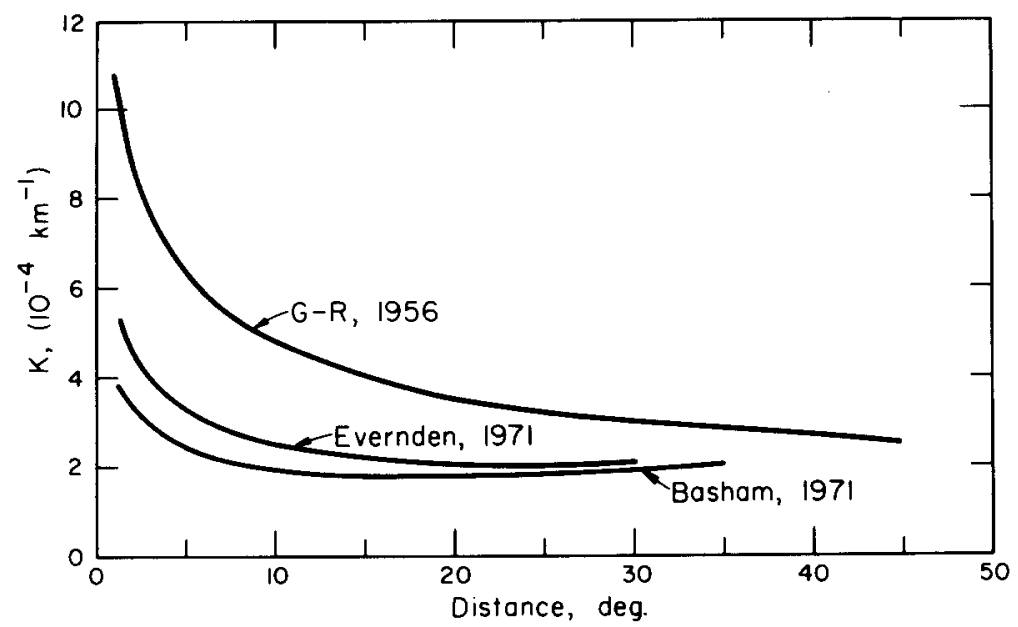

FIG. 1. Implied values of the energy-absorption coefficient, $k$, for three proposed empirical amplitudedistance correction equations.

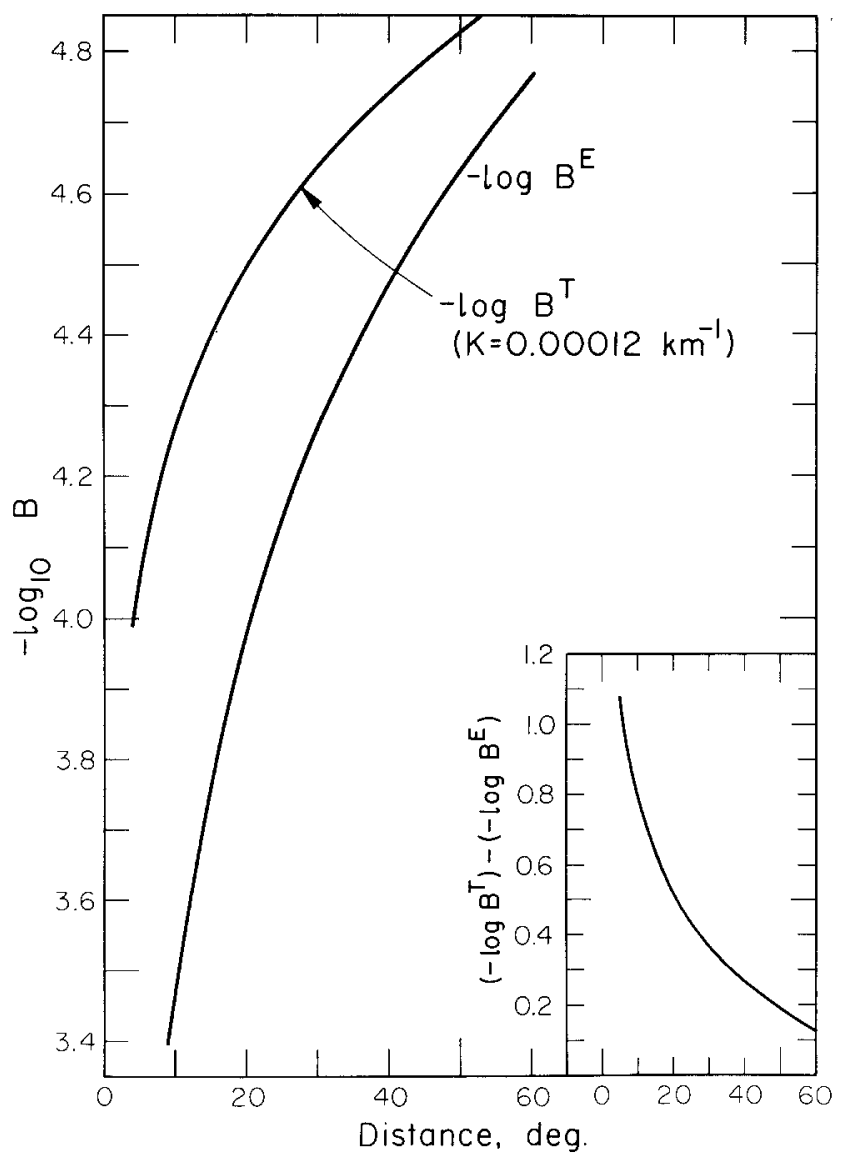

FYG. 2. Comparison of the empirical distance correction term, $-\log _{1_{0}} B^{E}$, and the theoretical term, $-\log _{10} B^{T},(11)$, for a continental-type absorption coefficient. The inset shows the difference between the two curves. 
If the simple linear Gutenberg-Richter relation is used to approximate the theoretical nonlinear term inside of the $15^{\circ}$ range, then the empirical term, $1.656 \log \Delta$, would incorrectly imply a distance dependence on $k$, and would result in absorption coefficient values much different from those observed for either continents or oceans. Figure 1 shows these implied coefficients calculated by equating (2) to (11) and solving for $k$ as a function of distance. From this figure, we see that by using the Gutenberg-Richter correction for distances less than $15^{\circ}$, the energy-dissipation coefficient of the medium implied by using the incorrect empirical formula in this range is much higher than that actually observed for either continents or oceans. This is just another way of expressing the fact that the linear empirical correction cannot approximate the nonlinear theoretical correction term with reasonable energy-dissipation coefficients for distances less than $15^{\circ}$. Also plotted on this figure are the implied $k$ 's for the empirical distance-correction factors proposed by Evernden (1971) and Basham (1971). As shown in this figure, the Evernden and Basham curves do indeed imply a satisfactory value of $k$ which is almost rangeindependent for distances greater than $4^{\circ}$ or $5^{\circ}$. It must be pointed out, however, that neither Evernden nor Basham included events with ranges less than $4^{\circ}$ in their leastsquares fit to get their empirical relations. This figure shows that perhaps still another empirical formula is needed to accurately estimate magnitudes from continental travel paths of less than $4^{\circ}$.

Now if we assume a continental energy dissipation factor of $1.2 \times 10^{-4} \mathrm{~km}^{-1}$, we see from Figure 2 that the empirical distance-correction factor is significantly lower than the theoretical correction for travel paths of less than $50^{\circ}$. For very short travel paths, the estimate of the surface-wave magnitude can differ by as much as one full unit. The inset to the figure shows the difference between the theoretical and the empirical values. The reason the two curves diverge as the travel path becomes smaller is the fact that the surface-wave magnitude scale normalizes amplitudes of a given type of travel path to the amplitude observed at $90^{\circ}$, not to that observed at the epicenter. If the surfacewave magnitude is calculated using the empirical formula, but the travel path is typically continental, then this difference shown in Figure 2 must be added to the magnitude using (2). It should be noted from this figure that even at a range of $20^{\circ}$, where the empirical correction is supposedly a good approximation to the observed data with oceanic travel paths, and also a good approximation to the theoretical term with an oceanic dissipation constant, there is still a half unit of magnitude difference between that given by the empirical correction and that calculated theoretically for a continental travel path.

Figure 3 shows the effect of adding this continental correction to the magnitudes derived from the empirical correction using long-period LRSM recordings of Nevada Test Site (NTS) events as given by Evernden (1971). At each of the stations where a magnitude was determined, the travel path was purely continental. Although this figure still indicates some scatter to the corrected magnitudes, there is no longer any apparent distance dependence on the magnitude derived for each event. Evernden (1971) also presents magnitude measurements found by applying (4) to continental earthquakes which also exhibit this same magnitude-distance dependence. In Figure 3, a dashed line has has been drawn to the best fit to each of the corrected magnitude data sets. In each case, the theoretical surface-wave magnitude for this particular continental energy-dissipation coefficient is slightly larger than that predicted by the asymptotic fit at large epicentral distances to the empirical formula. If an unreasonable energy-dissipation coefficient were chosen in the calculations, then a magnitude-distance relation would reappear in the corrected data. Therefore, to be rigorous in determining the surface-wave magnitude, the average energy-absorption coefficient for the travel path must be calculated and the magnitude determined at a particular station will be a function of this coefficient. 
In the above discussion, we have assumed one value for the energy-absorption coefficient and applied this to each magnitude determination regardless of the actual period used. For the Sedan event, the average value of the periods used in the magnitude determination at each individual station as given in the LRSM shot report for that event was about $16.5 \mathrm{sec}$. Mitchell (1972), in a study involving attentuation of Rayleigh waves in the central United States, gives an energy-decay coefficient of about $1.2 \times 10^{-4}$ $\mathrm{km}^{-1}$ for a period of $16.5 \mathrm{sec}$. This value is also a good average of the continental coefficients given by Gutenberg (1945) and Tryggvason (1965) for the period range 17 to $25 \mathrm{sec}$. Consequently, this was the value chosen to approximate the decay coefficient used in these continental magnitude determinations. It is easily seen from an evaluation

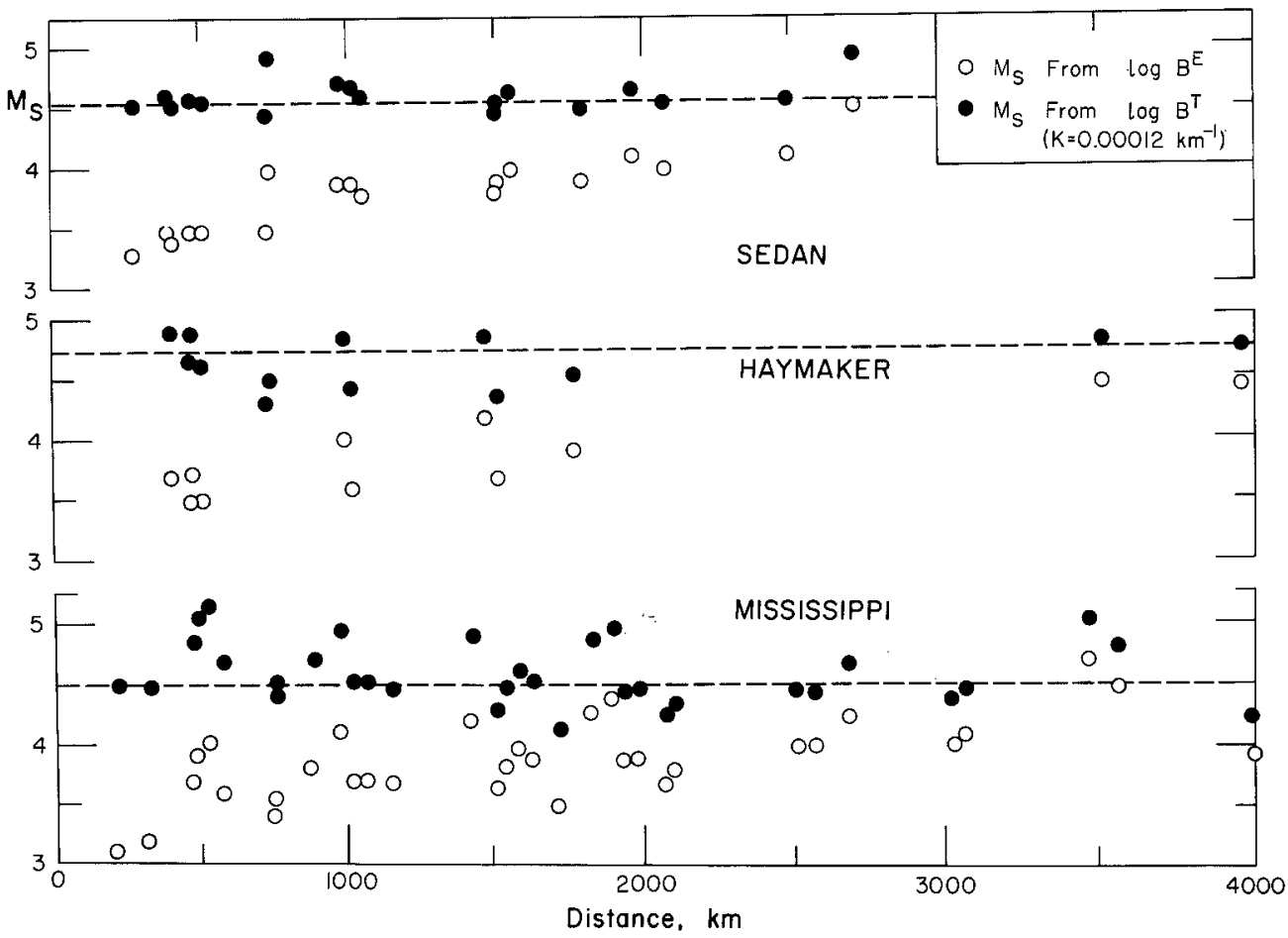

FIG. 3. Application of the theoretical distance correction factor, (11), with $k=0.00012 \mathrm{~km}^{-1}$, to NTS data. Magnitude values using $-\log _{10} B^{E}$ obtained from Evernden (1971).

of the contribution of the absorption coefficient to the theoretical distance correction term, that a slight error in the approximation of the path-averaged energy-dissipation coefficient will not adversely affect the resulting magnitude determination. Application of the appropriate path-averaged dissipation coefficient for the particular energy-arrival period used in the magnitude determination might help explain the dependence of surfacewave magnitude on period as reported by Evernden and Filson (1971).

Now using (11), it is possible to tabulate the correction factor $-\log _{10} B^{T}$ for different distances and absorption coefficients, so that this factor can be easily used in magnitude determinations. Table 1 gives $-\log _{10} B^{T}$ as expressed by equation (11). For comparison, the empirical values derived by (2) are listed in the last column of this table. The two variables in this table, $k$ and distance, are given in sufficiently small increments so that interpolation between values in the table can be done without any significant loss of accuracy. 
TABLE 1

Values of $-\log _{10} B^{T}$ Given by (11) for Various Values of $k$ and Distance

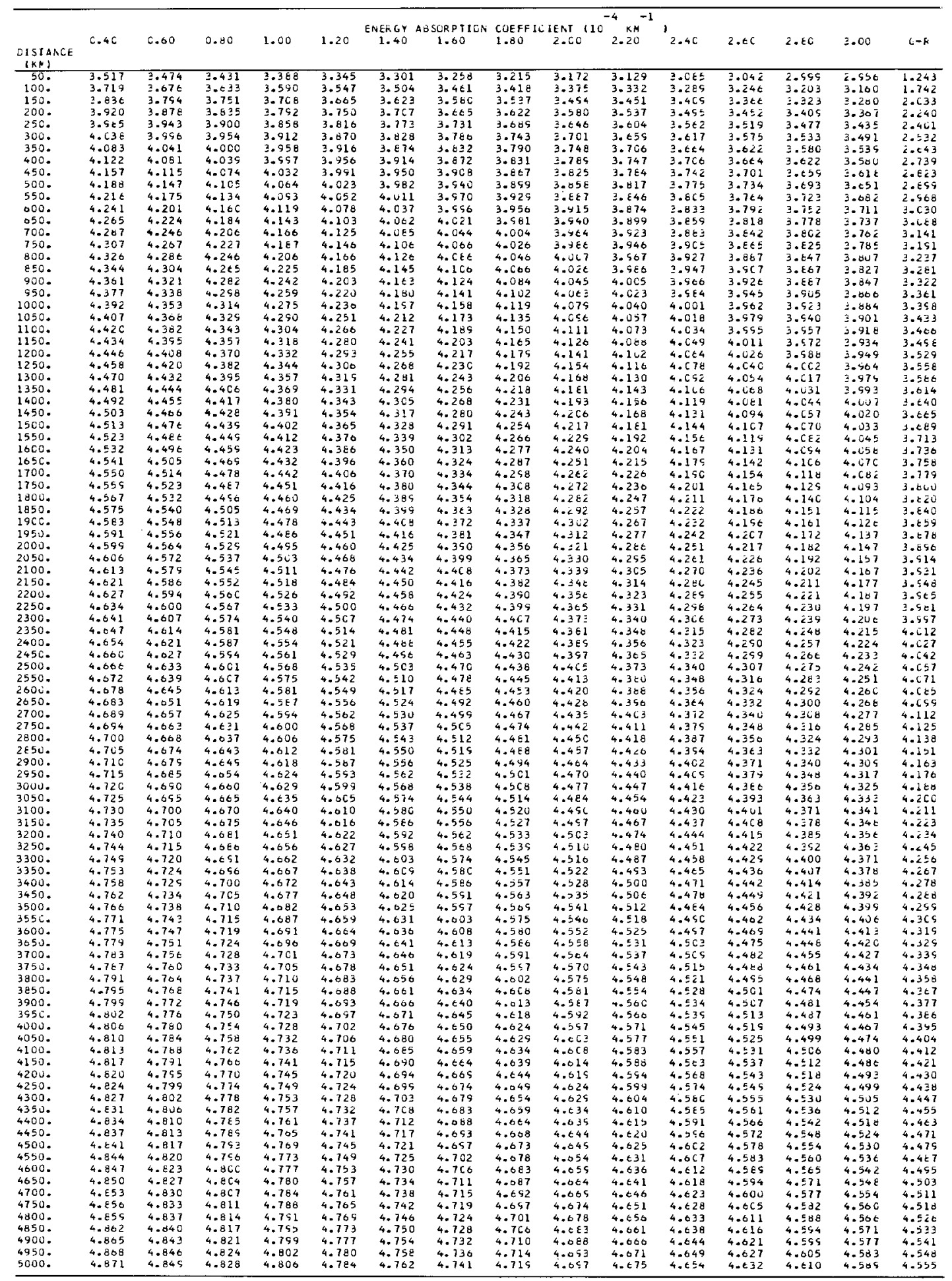


The application of this theoretical surface-wave magnitude formula to the earthquakeexplosion discrimination problem is immediate. Surface-wave magnitudes obtained for different propagation paths may be compared by merely normalizing the magnitudes obtained by using one particular path-averaged dissipation coefficient to that obtained by using some "standard" coefficient. This normalization might provide a means for better separating natural events and explosions in the $m_{b}-M_{s}$ plots.

This normalization can be accomplished by defining

$$
M_{s_{0}}=M_{s}(k)-N(k)
$$

where $N(k)$ is the normalization term given by

$$
N(k)=\log _{10}\left[\mathrm{e}^{r\left(k_{0}-k\right)} \cdot\right]=0.434 r\left(k_{0}-k\right)
$$

where $k_{0}$ is the "standard" energy dissipation coefficient, $k$ is the particular pathaveraged energy-dissipation coefficients of the period of the maximum amplitude, and $r$ is the source to receiver distance.

In conclusion, we observe that for measurements of surface-wave magnitude for events having purely continental travel paths, the widely accepted distance correction formula fails to give accurate consistent results, especially for short propagation distances. This empirical distance-correction term, which was never intended for general use for events having propagation paths less than $15^{\circ}$, implies energy-absorption coefficients higher than those observed over an average continental path. Calculations of magnitudes computed using a theoretical distance correction term, which takes into account both the absorption and dispersion properties of a typical continental structure, seem to give accurate, distance-independent results. Magnitudes computed using this theoretical formula can be normalized to take account of the average dissipation coefficient along the travel path, and, thus, provide a valid means to systematically compare magnitudes obtained for different events and travel paths.

\section{ACKNOWLEDGMENTS}

The author would like to thank Drs. David G. Harkrider and Otto W. Nuttli for their helpful comments made during this investigation, and Drs. Brian J. Mitchell and Otto W. Nuttli for making available their papers in advance of publication. This research was supported by the Advanced Research Projects Agency of the Department of Defense and was monitored by the Air Force Office of Scientific Research under Contract No. AF-F44620-70-C-0120, Contract No. AF-F44620-69-C-0067, and Contract No. AFOSR-70-1954.

\section{REFERENCES}

Alewine, R. W. (1972). Dispersion effects on Rayleigh wave magnitude (in preparation).

Basham, P. W. (1971). A new magnitude formula for short period continental Rayleigh waves, Geophys. $J .23,255-260$.

Evernden, J. F. (1971). Variation of Rayleigh-wave amplitude with distance, Bull. Seism. Soc. Am. 61, 231-240.

Evernden, J. F., W. J. Best, P. W. Pomeroy, T. V. McEvilly, J. M. Savino, and L. R. Sykes (1971). Discrimination between small-magnitude earthquakes and explosions, J. Geophys. Res. 76, 8042-8055.

Evernden, J. F. and J. Filson (1971). Regional dependence of surface-wave versus body-wave magnitudes, J. Geophys. Res. 76, 3303-3308.

Ewing, W. M., W. S. Jardetzky, and F. Press (1957). Elastic Waves in Layered Media, McGraw-Hill Book Co., p. 358.

Gutenberg, B. (1945). Amplitudes of surface waves and magnitudes of shallow earthquakes, Bull. Seism. Soc. Am. 35, 3-12.

Gutenberg, B. and C. F. Richter (1936). On seismic waves, Beitr. Geophysik 47, 73-131.

Long Range Seismic Measurements Project 8.4, Sedan (1962). DATDC Report No. 36, United Electrodynamics Inc., Alexandria, Va. 
Mitchell, B. J. (1972). Radiation and attenuation of Rayleigh waves from the Southeastern Missouri earthquake of October 21, 1965, submitted to J. Geophys. Res.

Nuttli, O. W. (1972). Seismic wave attenuation and magnitude relations for eastern North America, submitted to $J$. Geophys. Res.

Richter, C. F. (1958). Elementary Seismology, W. H. Freeman and Co., San Francisco, p. 346.

Taner, D. (1961). Equation de magnitude pour la station seismologique d'Istanbul-Kandilli concernant les seismes prouches, non profounds $(h<60 \mathrm{~km}$ ), Milli Egitim Bakanligi 6, 1-8.

Tryggvason, Eysteinn (1965). Dissipation of Rayleigh-wave energy, J. Geophys. Res. 70, 1449-1455.

Tsai, Y. and K. Aki (1969). Simultaneous determination of the seismic moment and attentuation of seismic surface waves, Bull. Seism. Soc. Am. 59, 275-287.

Vanek, J., A. Zatopek, V. Karnik, N. V. Kondorskaya, Yu. V. Riznichenko, E. F. Savarensky, S. L. Solov'ev, and N. V. Shebalin (1962). Standardization of magnitude scales, Bull. Acad. Sci. USSR, Geophys. Ser. 2, 108-111.

SEISMOLOGICAL LABORATORY

California Institute of Technology

Pasadena, California 91109

Division of Geological and Planetary Sciences

Contribution No. 2088

Manuscript received November 8, 1971 